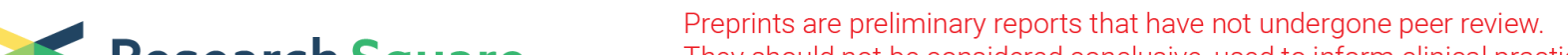 Research Square They should not be considered conclusive, used to inform clinical practice, or referenced by the media as validated information.
}

\section{Intrinsic Network Brain Dysfunction Correlates With Temporal Complexity in Post-traumatic Stress Disorder}

\section{Yingliang Dai}

Nanjing Normal University

\section{Zhou Zhou}

Nanjing Normal University

\section{Feng Chen}

Hainan General Hospital

\section{Li Zhang}

Second Xiangya Hospital

\section{Jun Ke}

The First Affiliated Hospital of Soochow University

\section{Rongfeng Qi}

Nanjing Jinling Hospital: East Region Military Command General Hospital

\section{Guangming Lu}

Nanjing Jinling Hospital: East Region Military Command General Hospital

Yuan Zhong ( $\nabla$ zhongyuan@njnu.edu.cn )

Nanjing Normal University School of Psychology https://orcid.org/0000-0002-4237-3206

\section{Research Article}

Keywords: Post-traumatic stress disorder, dynamic functional connectivity, neural networks

Posted Date: December 28th, 2021

DOI: https://doi.org/10.21203/rs.3.rs-1151746/v1

License: (c) (1) This work is licensed under a Creative Commons Attribution 4.0 International License. Read Full License 


\section{Abstract}

\section{Background}

Research has been looking into neural pathophysiology of post-traumatic stress disorder (PTSD) and dynamic functioning connectivity ( $\mathrm{dFC}$ ) applying resting state functional magnetic resonance imaging (rs-fMRI). Previous studies showed that PTSD related impairments are associated with alterations distributed across different brain regions and disorganized functional connectivity, especially in Default Mode Network and the cerebellar area. In this study, we specifically looked into dFC on a whole brain level, and we focused on critical regions such as DMN and cerebellum.

Methods

To explore the characteristics of dFC among patients with PTSD, we collected rs-fMRI data from 27 PTSD patients and 30 healthy controls. The study also added a control group of 33 trauma-exposed individuals to further look into trauma impact. Utilizing group spatial independent component analysis (ICA), the dynamic properties on whole brain level were detected with sliding time window approach, and k-means clustering.

Results

Two reoccurring FC "States" were identified, with connections being more concentrated on a withinnetwork level in one state and more strongly inter-connected in the other state. Abnormalities in dFC were found within DMN, between DMN and cerebellum, and between DMN and visual network.

\section{Conclusions}

The findings were in accordance with the study hypothesis that PTSD patients demonstrated deficits in emotional modulation and dysfunctional self-referential thoughts, and the deficits could be indicated in $\mathrm{dFC}$ alterations. Abnormalities in dFC among PTSD patients could serve as appropriate indicators of PTSD symptoms including depression and anxiety, hypervigilance, as well as impaired cognitive functioning and self-referential information processing.

\section{Introduction}

Post-traumatic stress disorder (PTSD) is prevalent in population exposed to traumatic events and its persisting negative impact on quality of life [1,2]. According to a cross-national study, the prevalence of PTSD reached $5.6 \%$ among population exposed to traumatic events, and the prevalence rate among general population has reached approximately 3.6\% [3]. More than half of PTSD patients remain untreated [3], which has become a public health concern given the high suicide risk and related substance abuse [4]. Symptoms of PTSD include hyperarousal, re-experiencing, avoidance, and negative or numb emotional state $[4,5]$. Impairments in fear processing, cognitive functioning and emotional modulation are recognized to be associated with PTSD, which contribute to the chronicity of symptoms $[1,6]$. Barriers 
still exist in identifying and treating PTSD, hence study of neural physiopathology is essential in further facilitating diagnosis and treatment of this psychiatric disorder [7].

Multiple previous studies have demonstrated that PTSD related impairments are associated with alterations in brain regions, as well as with disorganized functional connectivity $[8,9]$. The assumption in previous neuroimaging studies has been that fluctuations were static intrinsically during the entire recording period $[10,11]$

Resting state networks (RSNs) has become a research focus in recent neural physiopathology studies, using resting-state functional magnetic resonance imaging (rs-fMRI) [10,12]. The measurement of RSfMRI serves to demonstrate the state of brain activity across brain areas by detecting how of blood oxygen level-dependent (BOLD) signals were organized with no stimuli $[13,14,15]$. RSNs are thus made available to be observed through rs-fMRI and be assessed to examine potential abnormalities within and between different networks [16]. With regards to PTSD, much attention has been paid to RSNs, especially default mode network (DMN). DMN mainly anchors posterior cingulate cortex (PCC), mPFC, precuneus, and lateral temporal cortices $[17,18]$, and is an important network in self-related information processing and emotional regulation. Disruptions in DMN are demonstrated to be related to PTSD symptoms including difficulty regulating emotions and intrinsic thoughts [1]. In addition, cerebellum as a brain region that has been understudied in psychiatric disorders is also shown to play a critical role in PTSD symptomology. The altered functional connectivity between cerebellum and other regions such as prefrontal regions is found to be associated with bodily consciousness and multisensory integration [19]. There is growing research interest in dynamic analysis instead of static analysis of brain network connectivity on discrete level.

Latest research showed that dynamic alternations of functional connectivity (dFC), especially temporal variability, could serve as indication of changes in patterns of neural activity on a macro level [11]. Therefore, in this aspect, $\mathrm{dFC}$ compared to static FC could reveal more time variance features, and provide more precise biomarkers of psychiatric disorders like PTSD [20]. The approach of group independent component analysis, in the meantime, could be used to decompose the whole brain $\mathrm{mRI}$ data into distinct functional regions [11]. This approach could help resolve the issue of merging areas when applying regions of interest based atlas [11].

In our study, we explored potential differences in dFC among PTSD patients, trauma exposed individuals without PTSD diagnosis, and healthy individuals. One hypothesis is that individuals with PTSD tend to demonstrate alterations in dynamic functional connectivity in comparison to healthy individuals, and trauma exposed individuals would demonstrate similar alterations but to a less significant extent. Another hypothesis is that the alterations would be direct indicators of exposure to trauma and predictor of level of trauma impact. Finally, it was also stipulated that trauma related alterations in functional connectivity would correlate with and predictive of PTSD symptoms, including emotional symptoms, impaired cognitive functioning and memory processing. 


\section{Materials And Methods}

\section{Materials and Methods}

In $2014,18^{\text {th }}$ of July, a tropical category 4 typhoon named Rammasun severely affected Wenchang city in Hainan province located in southern China. At least 14 deaths were reported as the consequence of the disaster, and it was an especially hard hit on individuals dwelling locally. In addition, in Luodou town, which is part of Wenchang city, a thousand individuals experienced being trapped by storm tide subsequent to the destructive natural event. 70 individuals affected by the typhoon in the surrounding area were recruited, and among these individuals, 36 were diagnosed with PTSD including nine males and twenty-seven females. The other group of 34 were without PTSD (TECs, 7 males and 27 females). Recruited subjects all went through screening with the PTSD Checklist-Civilian Version (PCL). Diagnostic criteria specified in DSM IV was applied for diagnosing PTSD. Clinician-Administered PTSD Scale (CAPS) were applied to assess clinical symptoms $[10,21]$. Information is obtained through the scale regarding duration, symptom onset, and impact on functioning. Presence or absence of comorbid disorders was determined by applying the Structural Clinical Review for DSM IV. Additionally, 32 healthy controls were recruited, including nine males and twenty-three females. These individuals didn't meet diagnostic criteria for PTSD, and the subjects were recruited from Haiko, a city about 35 kilometers away from Wenchang. Assessment of depression and anxiety symptoms was conducted administering Self-Rating Depression Scale (SDS) and Self-Rating Anxiety Scale (SAS). The whole process lasted from November 2014 to January 2015.

The following were the applied general exclusion criteria: (a) age under eighteen or above sixty-five; (b) significant neurological and medical conditions; (c) history of head injury or loss of consciousness; (d) left handedness; (e) current comorbid or lifetime comorbid psychiatric disorders other than depression and anxiety; ( $f$ ) psychotropic medication use (g) alcohol or substance abuse; (h) contraindications for MRI, such as pregnancy, claustrophobia or ferromagnetic implants [22]. Completed imaging data was unavailable for 3 female participants in PTSD group, and 6 other participants were excluded due to brain infarction ( 1 female), denture-related artifact ( 1 female, 1 male), pregnancy ( 1 female), excessive head movement (1 female, 1 male). Two male participants in HC group were excluded due to brain infarction and 1 female participant was excluded due to excessive head movement. Eventually, 27 PTSD subjects, 33 TEC subjects and $30 \mathrm{HC}$ subjects participated in the study.

The study was conducted according to declaration of Helsinki. Approval was provided by committee of ethics in the Second Xiangya Hospital of Central South University and Hainan General Hospital has provided approval. Signed consent was collected from all participating subjects after being informed of the study description.

\section{Data Acquisition}

The resting-state magnetic resonance imaging scans were completed with the use of 3 Tesla MRI scanner (Skyra, Siemens Medical Solutions, Erlangen, Germany), standard 32 channel head coil [4]. 
Participating subjects received instructions to close their eyes, lie still and not fall asleep. High resolution T1-weighted 3D images were captured with a sagittal magnetized, single shot, rapid gradient-recalled sequence of echo to co-register and normalize subsequently. The repetition time or echo time equals $2300 / 1.97 \mathrm{~ms}$, with the flip angle equaling $9^{\circ}$. FOV equals $256 \times 256 \mathrm{~mm} 2$, matrix equals $256 \times 256$, total 176 slices, with slice thickness equaling $1 \mathrm{~mm}$. The scanning lasted 500 seconds for each MRI. Restingstate $\mathrm{fMRI}$ scans of the whole brain were obtained with the use of gradient-echo planar imaging (Total volumes equaled 250 , TR/TE equaled $2000 / 30 \mathrm{~ms}$, flip angle equaled $90^{\circ}$, FOV equaled $230 \times 230 \mathrm{~mm} 2$, matrix equaled $64 \times 64,35$ slices and no intersection gap, slice thickness $=3.6 \mathrm{~mm}$ ). Anterior-posterior commissure was referred to for parallel alignment.

\section{Data preprocessing}

Data was processed with the use of toolbox Data Processing and Analysis of Brain Imaging (DPABI) (http://rfmri.org/dpabi), which was run in MATLAB (Mathworks Inc., Sherborn, MA, USA) [23].

Realignment of data to the first volume was carried out for the purpose of correcting head motions. Data was further assorted into gray matter, white matter and cerebrospinal fluid with the use of the Tissue Probability Map template. Subsequently, the normalization matrix acquired was used to smooth the functional images spatially with the convolutional effect of an isotropic Gaussian kernel of $6 \mathrm{~mm}$.

Corrective measures were performed on head motion post realignment. Exclusion of subjects with head motion exceeding $1.0 \mathrm{~mm}$ or rotation exceeding $1.0^{\circ}$ when scanned was performed. Group differences were evaluated with the formula: Head Motion/Rotation $=\frac{1}{L-1} \sum_{i=2}^{L} \sqrt{\left|x_{i}-x_{i-1}\right|^{2}+\left|y_{i}-y_{i-1}\right|^{2}+\left|z_{i}-z_{i-1}\right|^{2}} \quad: L$ being the time series length and in current study L=240 [24]. It was indicated in the results that the two groups were not significantly different in quality of image (One-way ANOVA, $F=1.39, p=0.255$ for translational motion, and $F=0.125, p=0.883$ for rotational motion).

\section{Group independent component analysis (ICA)}

Following data preprocessing, group spatial ICA was conducted with the GIFT toolbox based on Matlab 2020 a $[25,26]$ for data to be decomposed into functional networks.

Data reduction was conducted with application of principle component analysis. At subject-specific data level, independent components (ICs) were reduced to 120 with the application of principal components analysis. Group ICs were further decreased to 100 at group level, with the expectation-maximization algorithm $[27,28]$. The ICA algorithm was repeatedly run for 20 times in ICASSO [27®29] in order to ensure the infomax ICA algorithm stability and reliability [30].

We subsequently clustered components that resulted from last step. We conducted manual confirmation of peak activation coordinates and examined if they were distributed in grey matter primarily [31]. Approximately 34 relevant components were selected based on the previous procedures for estimating 
reliability. Via back-reconstruction approach (GICA) we obtained subject-specific spatial maps, as well as time courses [32].

Related intrinsic connectivity networks were identified as a result of the described procedures. In addition, confirmation of high to low frequency fluctuation ratio and whether peak activation coordinates were mainly grey matter located was made to eventually narrow the ICs to 34 .

\section{Dynamic functional connectivity}

\section{Sliding window approach}

There was growing application of sliding time window approach in research for investigating DFC, and we used this approach in the study to look at time-varying changes in FC [11, 27, 33, 34]. Resting state data was segmented and resulted in windows of 22 repetition times with a size of $44 \mathrm{~s}$. It has been demonstrated that this segment length well balanced the ability to resolve dynamics and correlation matrix estimation quality [27]. A window length of 30 to 60 seconds was identified consequently, and topological assessments of brain networks were stabilized at around 30 seconds [27]. The window length was restricted with a sigma 3-TR of Gaussian, one repetition time [33]. Above mentioned steps resulted in 218 windows that consecutively distributed across the whole scan. In order to encompass all possible pairs of the 34 ICs selected within each window, we calculated 34 x 34 pair-wise covariance matrix. In addition, the L1 norm was used in LASSO framework in order to promote sparsity in estimation with 100 repetitions $[33,35]$. The resulting values were transformed into z-scores, which was achieved through Fisher's z transformation to reduce variance effect. Eventually, the matrices that went through ztransformation were residualized with nuanced variables including gender and age [31].

\section{Clustering analysis}

The clustering method of k-means was applied for clustering the 218 window FC matrices obtained from last analysis step, with the goal to identify reoccurring FC states. The L1 distance (Manhattan distance) was employed to measure the similarity of FC matrices between windows, since it was more effective compared to L2 distance when being applied on high-dimension data [11]. According to Allen et al. [11], when performing subsampling of windows, a result of approximately 2 windows for each subject was produced. Therefore, through silhouette, the cluster number of 2 was set. The optimal cluster number was tested again through repeating the analysis with other set number, however almost identical clusters were produced and lacked significant distinction. The cluster number of 2 was also widely applied in previous literature $[27,33,36]$. All FC matrices were clustered into either state I or state II. The calculated medians of the FC matrices were computed as the centroids of two clusters. The k-means algorithm was repeated for 100 times to reduce potential random selection bias. The clustering analysis procedures were performed on all subjects as a whole group, and on the three sub-groups separately. The purpose of this step was to compare the differences among the three groups in terms of the connectivity pattern as well as connection strength of the two states. Permutation one-way analysis of variance (ANOVA) was applied for state comparison among the three groups ( $p<0.01$, FDR corrected). 


\section{State analysis}

In order to explore the temporal properties of the two dFC states, we obtained the comparison of number of state transitions, fractional windows, and mean dwell time among the three groups (HC, PTSD, TEC). This analysis was based on the previous clustering analysis results, and therefore the temporal properties of the three groups were calculated separately. The temporal properties of the whole group were also calculated, however the results were not used since it was not meaningful for this study. For "fractional windows", the measurement was number of windows in one state. For "mean dwell time", the measurement was average amount of consecutive windows in one state before shifting into the other state. For "number of transitions", it stood for literal meaning which was the times these three groups switched from one state to the other, indicating reliability of each state. Since there were three groups of subjects, ANOVA was applied to examine between-group differences among healthy controls, PTSD subjects and trauma-exposed subjects, with year of education, age and gender as covariates $(p<0.01$, FDR corrected).

\section{Statistical comparisons and correlations analysis}

We applied ANOVA for comparison of the significance of differences among demographic and clinical characteristics $(\mathrm{p}<0.001)$. We compared the three groups in pairs, and gained the $p$ values for the three sets of comparison. The analysis was performed via SPSS Statistic, release version 26.0 (Chicago, IL, USA) .

In order to examine correlations between SDS and SAS scores of clinical scales and altered network temporal properties, Spearman's correlation analysis was performed in PTSD and control group $(p<0.05$, uncorrected). We also performed correlational analysis in PTSD group between CAPS and IES scores and altered network temporal properties. We applied SPSS to perform all statistical analysis.

\section{Results}

\section{Demographic and clinical characteristics}

Table 1 presented the results of analysis for clinical and demographic characteristics. There was no significant difference among control, PTSD and trauma exposed groups in gender distribution $(p=0.912)$ or in age $(F=0.317, p=0.729)$. It was found that the three groups were significantly different on education level $(F=8.396, p<0.001)$. In addition, it was demonstrated that the SAS as well as SDS scores in PTSD group is significantly higher compared to TEC and HC groups $(p<0.001)$, while the scores of HC group are the lowest among the three groups. The PCL scores of PTSD group is higher than those of trauma exposed groups $(p<0.001)$ significantly. The mean scores of CAPS among PTSD patients is $78.2 \pm 19.3$.

\section{Intrinsic connectivity networks}

We grouped all identified 34 independent components into seven intrinsic connectivity networks and one region - the cerebellar region - on the basis of the functional and anatomical properties. The networks are 
as followed: basal ganglia (BG), Auditory Network (AUD), Visual Network (VIS), Sensory-Motor Network $(\mathrm{SMN})$, Central Executive Network, Default Mode Network (DMN), SN, and cerebellum (CM). Here, cerebellum refers to the cerebellar region. Spatial maps of the selected ICs are presented in Figure 1.

\section{Dynamic functional connectivity state analysis}

\section{Temporal properties}

K-means analysis resulted in two recurring functional connectivity states. The resulting State I is more sparsely connected, and State II is more strongly interconnected in comparison. Among all the participants, State I (79\%) occurrence is more frequent than State II (21\%). As shown in Figure 2A and B, the two functional connectivity state patterns are visually different in distribution of connectivity strength.

As presented in Figure $3 \mathrm{~A}$ and $\mathrm{B}$, differences were observed when looking at group-specific centroid of clusters. In State I, connections were segregated and were mainly concentrated within DMN and CEN networks. It was also observed that the connection between DMN and CEN was negative, which indicated that DMN was negatively correlated with CEN in resting state. State II on the other hand demonstrated stronger interconnectivity between networks. In addition, more within-network connections were active in State II, including AUD, VIS, SMN, SN, CB, CEN and DMN. The interconnections involved the AUD, VIS, BG, $\mathrm{SMN}$, and $\mathrm{CM}$. State II was also a state where large scale brain connections were stronger and more interactive since both CEN and DMN were activated. The CEN-AUD, CEN-VIS, CEN-SMN connections were among the most salient connections in state II, while DMN and SN also demonstrated positive connections with other networks. Notably, BG and CB demonstrated mostly negative connections with other networks in this state.

It was shown in Figure 4A that State I occurred more frequently for all three groups of participants. In addition, compared to control group, State I occurred significantly more frequent in PTSD group $(p=0.026)$. Meanwhile, it was observed that there was more frequent occurrence in State I for TEC group compared to control group, but PTSD had the most frequent occurrence in State I among the three groups.

Figure 4B visually illustrated mean dwell time for the three groups. It was concluded that PTSD patients dwelled significantly longer in the less activated and connected state $(p=0.0094)$, in comparison with HC group. The difference of dwell time in State II between HC and PTSD group was also statistically significant $(p=0.0075)$. In addition, the trauma-exposed participants compared to control group generally remained longer in State I.

With regards to number of transitions, healthy controls were shown to transit the most frequently between two states, in comparison to PTSD and TEC groups, and the transition frequency of healthy controls was found to be higher than PTSD group $(p<0.05)$. Among the three groups, the transition frequency was the lowest in PTSD group among the three groups. The result is presented in Figure 4C. 
Correlational analysis (Table 2) demonstrated significant correlations between temporal properties and clinical scores $(p<0.05)$. For SAS scores which indicated anxiety symptom, significant correlation was observed with State I mean dwell time. For SDS scores which indicated depressive symptom, significant correlations were found with State I and State II mean dwell time, and fractional windows. For symptom of hypervigilance, significant correlations were found with State I mean dwell time and number of transitions. Notably, among all significant correlations, two negative correlations were identified: correlation between dwell time in State II and SDS scores and correlation between number of transitions and hypervigilance.

\section{Strength of dynamics states}

Based on the described methods, connection strength of the two states was compared among the three groups. Since the difference was not significant for TEC group, only the comparison between HC and PTSD group was further discussed. In state I, 6 within- and between-network connections were identified that were stronger among healthy controls (HC>PTSD, $p<0.01$, corrected). Notably, for control group >PTSD group connections, $100 \%$ of them (6/6) were related to DMN network, including within-DMN, DMN-VIS and DMN-CB network connections. We found 1 between-network connection that was stronger among the PTSD group (HC<PTSD, $p<0.01$, corrected). which was consistent with the result of State I. The connection was located within CEN-SN network. The same analysis was repeated for State II. When comparing healthy controls to PTSD patients, there was only 1 between-network connection that was stronger among the PTSD group (HC<PTSD, $p<0.01$, FDR corrected).

\section{Discussion}

In the past few years, research has been looking into the abnormalities on functional connectivity level for PTSD in order to identify diagnostic biomarkers. Dynamic functional connectivity during resting state has been proven by scholars to be effective in predicting neuropsychiatric disorders [11,37]. This is one of the first studies to look at whole brain level network functional connectivity during resting state using independent component analysis among research on PTSD. With the hope to further understand the neural physiopathology of PTSD and thus facilitate more precise diagnosis, we focused on the specific as well as common time-varying alterations of functional network connectivity.

\section{State Connectivity}

Two distinct reoccurring states were identified during the entire fMRI scan across all participants. It could be observed from our results that connections in State I were more concentrated on a within-network level, while state II being more strongly inter-connected. In addition, State II was a more strongly connected state in terms of the within-network connection strength. The different states demonstrated time-varying dynamic features of human brain activity during resting state. When conducting cross-group comparison, we observed significant differences between PTSD patients and healthy controls in the two states, which indicated that there existed alterations of dynamic functional connectivity among PTSD patients, and the alterations could serve as diagnostic markers of PTSD. 


\section{Temporal Properties}

As we observed in the study, PTSD group demonstrated significantly less flexibility when transitioning between two states, which was indicated by number of transitions. Despite that the differences between trauma exposed individuals and healthy controls were not statistically significant, the trend of decreased transitional flexibility as trauma impact level increase was illustrated by our results.

In addition, the PTSD group and TEC group also tended to remain longer in the less active, less strongly interconnected state, which is State I, when comparing to control group. It was stipulated that exposure to trauma might affect flexibility in functional connectivity, which could be a direct marker of cognitive functioning decline.

Based on previous studies, PTSD patients typically demonstrated lower variance in connectivity [38]. Especially, in State I, within-DMN connections were stronger, while between-network connections were weak and the whole brain connectivity was more segregated and less activated, which might be closer to a "baseline default state" [39]. Lower variance and less flexible transition might indicate that individuals with PTSD had already formed an altered default state where trauma was perseverating [38].

The perseverating effect of trauma could be further evidenced by the fact that trauma exposed subjects without PTSD also demonstrated less flexibility in transitioning between states compared to healthy controls. The results of analysis on temporal properties testified our hypothesis that individuals with PTSD tended to demonstrate alterations in dynamic functional connectivity, especially in terms of "baseline default state" with less flexibility and less active connectivity. Trauma exposed individuals demonstrated similar alterations but to a less significant extent.

\section{Correlational Analysis}

The Spearman correlation analysis resulted in significantly negative correlation between number of transitions and hypervigilance. This indicated that lower flexibility of state transition might have "primed" the symptoms such as hypervigilance and hyperarousal [38]. Taken together, the results indicated that trauma might affect baseline default state of human brain and the alteration might serve as basis of hypervigilant symptoms.

The correlational analysis also resulted in significantly positive correlation between dwell time in State I and emotional symptoms in PTSD group, including anxiety and depression. In addition, negative correlation between dwell time in State II and depression and anxiety was also observed. Increased dwell time in State I and decreased dwell time in State II was observed as trauma effect level increased, as both PTSD and TEC group dwell longer in State I compared to HC group. Combined with correlational analysis results, we stipulated that PTSD patients tended to remain longer in the weaker connected state, in which more depressive or anxiety symptoms might occur [36]. Trauma exposure was also stipulated to be associated with emotional symptoms, as TEC group also dwelled longer in the weaker connected state compared to HC group. This is consistent with previous studies regarding PTSD emotional 
symptomology. The results revealed that trauma related alterations in $\mathrm{dFC}$ were also predictive of emotional symptoms of PTSD, testifying our hypothesis.

\section{Between-group Comparison of Connectivity Strength}

In our study, connectivity strength was also analyzed, and there were significant differences between PTSD and health control group. The resulted significant differences in connectivity strength between healthy controls and PTSD patients were mainly related to DMN and cerebellar region, which was in accordance with previous literature. Scholars have pointed out that default mode network (DMN) is one of the most accurate networks in classifying PTSD patients, and serves as a significant biomarker [40]. Previous studies revealed disrupted resting state connectivity in DMN for patients diagnosed with PTSD $[1,41]$. It has been known that DMN is related to episodic memory and self-related information processing, and weaker connections in DMN could be associated with symptoms such as intrusive memories, ruminations and even dissociation $[1,10]$. In our studies, PTSD group demonstrated weaker connectivity strength within DMN network in State I, which was a baseline default state, in comparison to HC group. When compared with healthy controls, PTSD group also demonstrated weaker connection strength in DMN-cerebellum and DMN-VIS.

When looking into the specific independent components, it was shown that in a baseline default state, PTSD had weaker connection strength between left posterior cerebellum and left precuneus. In addition, weaker connections were also found among PTSD patients within the frontal lobe, and medial superior frontal gyrus also connected less strongly with interior paracingulate cortex as well as left occipital lobe. According to previous studies, weaker connection between left posterior cerebellum and bilateral precuneus could be indicative of state reliving and dissociative symptoms [42]. Individuals with PTSD also demonstrated weaker connections between cerebellum and frontal regions, which were associated with emotional regulation and awareness [42]. Considering the critical role posterior cerebellum plays in emotional regulation, the decrease of connectivity between this part and precuneus as well as frontal regions could evidence deficits in emotional modulation and dysfunctional self-referential thoughts among PTSD patients [43].

Similarly, altered connectivity between frontal lobe and occipital lobe also pointed to difficulty in processing of self-referential thoughts [44]. Finally, medial superior frontal gyrus and interior paracingulate cortex were also known to have association with cognitive functioning and self-referential information processing [5], and therefore the weaker connection could be associated with dysfunction in these two aspects. Therefore, this is another evidence suggesting that alterations in functional connectivity, especially in within- and between-network connection strength could be predictive of PTSD. The connectivity strength difference between PTSD group and HC group serves as predictive factor specifically in terms of cognitive functioning change and dissociative symptoms.

There are several limitations regarding the present study that should be noted and considered. Firstly, the sample size is small, especially when it comes to PTSD patients. Future study should consider a larger sample of PTSD patients in order to obtain more representative results. Second of all, this study is cross- 
sectional, and a more profound study could be conducted through tracking the cohort and applying longitudinal research methods. Finally, we only selected 34 independent components based on their relevance for the analysis. More independent components could be encompassed in order to obtain a more comprehensive and precise result.

\section{Conclusion}

Two distinctive states were identified during the entire fMRI scanning process, with State I being more segregated and less strongly connected and closer to a "baseline default state", and state II being more strongly interconnected with increase connectivity strength. It was found that subjects that were exposed to trauma, including TEC and PTSD subjects, demonstrated trend of longer dwell time in State I, which had correlation with emotional symptoms including depressive and anxious mood. Trauma exposed subjects also tended to transit less frequently between states, pointing to impairments in cognitive functioning. PTSD patients was found to demonstrate significantly weaker connectivity strength especially in DMN-cerebellum and DMN-VIS connections when compared with healthy controls. Specifically, weaker connectivity was found among PTSD patients between left posterior cerebellum and left precuneus, within the frontal lobe, between medial superior frontal gyrus and interior paracingulate cortex as well as left occipital lobe. The results of this study testified our hypothesis that individuals exposed to trauma demonstrated alterations in $\mathrm{dFC}$ compared to healthy individuals, and there were significant alterations when it comes to diagnosed PTSD patients. It was also proved that trauma related alterations in functional connectivity were predictive of PTSD symptoms, including emotional symptoms, impaired cognitive functioning and memory processing. This study evidenced that dynamic functional connectivity could serve as a diagnostic biomarker of PTSD especially in terms of deficits in cognition, emotional modulation, and dysfunctional self-referential thoughts.

\section{Declarations}

\section{Acknowledgements and Funding Sources}

This study was supported by the National Natural Science Foundation of China (81871344凶81971602, 81760308), the Natural Science Foundation of Jiangsu Province (BK20191369) and the Qing Lan project of higher education institutions of Jiangsu Province.

\section{Author Contributions}

Author Yingliang Dai conducted data analysis and drafted the article. Author Yuan Zhong is the corresponding author, provided guidance and instructions on data analysis and interpretation of results. Author Zhou Zhou conducted data analysis collaboratively with first author. Author Feng Chen recruited participants, collected and organized original data. Author Li Zhang performed screening and questionnaires for participants. Author Jun Ke performed fMRI data collection. Author Rongfeng Qi an 
author Guangming Lu performed fMRI data collection collaboratively. All authors have seen and approved of the final manuscript.

\section{Compliance with Ethical Standards}

The study was conducted according to declaration of Helsinki. Approval was provided by committee of ethics in the Second Xiangya Hospital of Central South University and Hainan General Hospital has provided approval.

\section{Conflict of Interest}

The authors report no financial interests or potential conflicts of interest.

\section{Consent to Participate}

Signed consent was collected from all participating subjects after being informed of the study description.

\section{Consent for Publication}

All authors have approved of publishing this manuscript which has been read thoroughly by all of them.

\section{Data Availability Statement}

The data that support the findings of this study involve human subjects, and are available on request from the corresponding author. The data are not publicly available due to privacy or ethical restrictions.

\section{Code Availability}

Not applicable.

\section{References}

1. Dretsch, M. N., Rangaprakash, D., Katz, J. S., Daniel, T. A., Goodman, A. M., Denney, T. S., \& Deshpande, G. (2019). Strength and temporal variance of the default mode network to investigate chronic mild traumatic brain injury in service members with psychological trauma. Journal of Experimental Neuroscience, 13,1-14

2. Thorp, S. R., \& Stein, M. B. (2005). Posttraumatic stress disorder and functioning. PTSD Res $Q, 16,1-$ 7

3. Koenen, K., Ratanatharathorn, A., Ng, L., McLaughlin, K., Bromet, E., Stein, D. ... Kessler, M., R (2017). Posttraumatic stress disorder in the World Mental Health Surveys. Psychological Medicine, 47(13), 2260-2274

4. Chen, H. J., Qi, R., Ke, J., Qiu, J., Xu, Q., Zhang, Z. ... Chen, F. (2020). Altered dynamic parahippocampus functional connectivity in patients with post-traumatic stress disorder. The world 
journal of biological psychiatry: the official journal of the World Federation of Societies of Biological Psychiatry, 1-10

5. Liu, T., Ke, J., Qi, R., Zhang, L., Zhang, Z., Xu, Q.... Lu, F.

6. Altered functional connectivity of the amygdala and its subregions in

7. typhoon-related post-traumatic stress disorder.Brain and Behavior, 11(1),e01952-e01967

8. Dretsch, M. N., Thiel, K. J., Athy, J. R., Irvin, C. R., Sirmon-Fjordbak, B., \& Salvatore, A. (2012). Mood symptoms contribute to working memory decrement in active-duty soldiers being treated for posttraumatic stress disorder. Brain and behavior, 2(4), 357-364

9. Chen, H. J., Qi, R., Ke, J., Qiu, J., Xu, Q., Zhong, Y. ... Chen, F. (2020). Evaluation of gray matter reduction in patients with typhoon-related posttraumatic stress disorder using causal network analysis of structural MRI. Psychological medicine, Sep 17, 1-10

10. Weng, Y., Qi, R., Chen, F., Ke, J., Zhong, Y., Chen, L. ... Lu, G. (2018). The temporal propagation of intrinsic brain activity associated with the occurrence of PTSD. Frontiers in Psychiatry, May Volume, $9,218-227$

11. Shalev, A., Liberzon, I., \& Marmar, C. (2017). Post-traumatic stress disorder. N Engl J Med, 376, 24592469

12. Ke, J., Zhang, L., Qi, R., Xu, Q., Zhong, Y., Liu, T. ... Chen, F. (2018). Typhoon-related post-traumatic stress disorder and trauma might lead to functional integration abnormalities in intra- and interresting state networks: a resting-state fmri independent component analysis. Cellular Physiology and Biochemistry, 48, 99-110

13. Allen, E. A., Damaraju, E., Plis, S. M., Erhardt, E. B., Eichele, T., \& Calhoun, V. D. (2014). Tracking wholebrain connectivity dynamics in the resting state. Cerebral cortex (New York, N.Y.: 1991), 24(3), 663676

14. Van Den Heuvel, M. P., \& Pol, H., H. E (2010). Exploring the brain network: a review on resting-state fMRI functional connectivity. European neuropsychopharmacology: the journal of the European College of Neuropsychopharmacology, 20(8), 519-534

15. Du, M., Zhang, L., Li, L., Ji, E., Han, X., Huang, G. ... Zhang, Z. (2021). Abnormal transitions of dynamic functional connectivity states in bipolar disorder: A whole-brain resting-state fMRI study. Journal of affective disorders, 289, 7-15

16. Fu, X., Liu, F., Cui, Z., \& Guo, W. (2020). Editorial: dynamic functional connectivity in neuropsychiatric disorders: methods and applications. Frontiers in Neuroscience, April Vol. 14, 1-3

17. Biswal, B. B., Van Kylen, J., \& Hyde, J. S. (1997). Simultaneous assessment of flow and BOLD signals in resting-state functional connectivity maps. NMR Biomed, 10, 165-170

18. Wang, D., Qin, W., Liu, Y., Zhang, Y., Jiang, T., \& Yu, C. (2014). Altered resting-state network connectivity in congenital blind. Human brain mapping, 35(6), 2573-2581

19. Kluetsch, R. C., Ros, T., Théberge, J., Frewen, P. A., Calhoun, V. D., Schmahl, C. ... Lanius, R. A. (2014). Plastic modulation of PTSD resting-state networks and subjective wellbeing by EEG neurofeedback. 
Acta psychiatrica Scandinavica, 130(2), 123-136

20. Raichle, M. E., Macleod, A. M., Snyder, A. Z., Powers, W. J., Gusnard, D. A., \& Shulman, G. L. (2001). A default mode of brain function. Proceedings of the National Academy of Sciences, 98(2), 676-682

21. Holmes, S. E., Scheinost, D., DellaGioia, N., Davis, M. T., Matuskey, D., Pietrzak, R. H. ... Esterlis, I. (2018). Cerebellar and prefrontal cortical alterations in PTSD: structural and functional evidence. Chronic stress (Thousand Oaks, Calif.), 2, 1-11

22. Keilholz, S. D. (2014). The neural basis of time-varying resting-state functional connectivity. Brain connectivity, 4(10), 769-779

23. Weathers, F. W., Keane, T. M., \& Davidson, J. R. (2001). Clinician-administered PTSD scale: a review of the first ten years of research. Depression and anxiety, 13(3), 132-156

24. Chen, H. J., Zhang, L., Ke, J., Qi, R., Xu, Q., Zhong, Y. ... Chen, F. (2019). Altered resting-state dorsal anterior cingulate cortex functional connectivity in patients with post-traumatic stress disorder. The Australian and New Zealand journal of psychiatry, 53(1), 68-79

25. Zhu, Y., Huang, M., Zhao, Y., Pei, Y., Wang, Y., Wang, L. ... Zeng, X. (2020). Local functional connectivity of patients with acute and remitting multiple sclerosis: A Kendall's coefficient of concordance- and coherence-regional homogeneity study. Medicine, 99(43), e22860

26. Chen, Y., Wang, C., Zhu, X., Tan, Y., \& Zhong, Y. (2015). Aberrant connectivity within the default mode network in first-episode, treatment-naïve major depressive disorder. Journal of affective disorders, $183,49-56$

27. Erhardt, E. B., Rachakonda, S., Bedrick, E. J., Allen, E. A., \& Adali, T., \&Calhoun

28. V. D. Comparison of multi-subject ICA methods for analysis of $\mathrm{fMRI}$

29. data.Human Brain Mapp, 32(12),2075-2095

30. Calhoun, V. D., Adali, T., Pearlson, G. D., \& Pekar, J. J. (2002). A method for

31. making group inferences from functional MRI data using independent component

32. analysis. Human Brain Mapping, 16(2),131-131

33. Fiorenzato, E., Strafella, A. P., Kim, J., Schifano, R., Weis, L., Antonini, A., \& Biundo, R. (2019). Dynamic functional connectivity changes associated with dementia in Parkinson's disease. Brain: a journal of neurology, 142(9), 2860-2872

34. Roweis, S. T. (1998). EM algorithms for PCA and SPCA. Advances in neural information processing systems. Cambridge: (pp. 626-632). MIT Press

35. Bell, A. J., \& Sejnowski, T. J. (1995). An information-maximization approach to blind separation and blind deconvolution. Neural Computation, 7(6), 1129-1159

36. Himberg, J., Hyvärinen, A., \& Esposito, F. (2004). Validating the independent components of neuroimaging time series via clustering and visualization. Neurolmage, 22(3), 1214-1222

37. Allen, E. A., Erhardt, E. B., Damaraju, E., Gruner, W., Segall, J. M., Silva, R. F. ... Calhoun, R. J., V. D (2011). A baseline for the multivariate comparison of resting-state networks. Frontiers in systems neuroscience, 5,2 
38. Calhoun, V. D., Adali, T., Pearlson, G. D., \& Pekar, J. J. (2001). Spatial and temporal independent component analysis of functional MRI data containing a pair of task-related waveforms. Human brain mapping, 13(1), 43-53

39. Kim, J., Criaud, M., Cho, S. S., Díez-Cirarda, M., Mihaescu, A., Coakeley, S. ... Strafella, A. P. (2017). Abnormal intrinsic brain functional network dynamics in Parkinson's disease. Brain: a journal of neurology, 140(11), 2955-2967

40. Damaraju, E., Allen, E. A., Belger, A., Ford, J. M., Mcewen, S., Mathalon, D. H., et al. (2014). Dynamic functional connectivity analysis reveals transient states of dysconnectivity in schizophrenia. Neuroimage Clin, 5, 298-308

41. Friedman, J., Hastie, T., \& Tibshirani, R. (2008). Sparse inverse covariance estimation with the graphical lasso. Biostatistics (Oxford, England), 9(3), 432-441

42. Yao, Z., Shi, J., Zhang, Z., Zheng, W., Hu, T., Li, Y. ... Hu, B. (2019). Altered dynamic functional connectivity in weakly-connected state in major depressive disorder. Clinical Neurophysiology, 130(11), 2096-2104.

43. Nieuwhof, F., \& Helmich, R. C. (2017). Entangled cerebral networks in Parkinson's disease. Brain, 140, 2767-2769

44. Jin, C., Jia, H., Lanka, P., Rangaprakash, D., Li, L., Liu, T. ... Deshpande, G. (2017). Dynamic brain connectivity is a better predictor of PTSD than static connectivity. Human brain mapping, 38(9), 4479-4496

45. Power, J. D., Schlaggar, B. L., \& Petersen, S. E. (2014). Studying brain organization via spontaneous fMRI signal. Neuron, 84, 681-696

46. Nicholson, A. A., Harricharan, S., Densmore, M., Neufeld, R., \& Ros, T., McKinnon

47. Frewen, M. C., Théberge, P. A., Jetly, J., \& Pedlar, R. (2020). D., Lanius, R. A.

48. Classifying heterogeneous presentations of PTSD via the default mode, central

49. executive, and salience networks with machine learning.Neurolmage: Clinical, 27, 102262 (1-15)

50. Koch, S. B., van Zuiden, M., Nawijn, L., Frijling, J. L., Veltman, D. J., \& Olff, M. (2016). Aberrant restingstate brain activity in posttraumatic stress disorder: a meta-analysis and systematic review.

Depression and anxiety, 33(7), 592-605

51. Rabellino, D., Densmore, M., Théberge, J., McKinnon, M. C., \& Lanius, R. A. (2018). The cerebellum after trauma: Resting-state functional connectivity of the cerebellum in posttraumatic stress disorder and its dissociative subtype. Human brain mapping, 39(8), 3354-3374

52. Verger, A., Rousseau, P. F., Malbos, E., Chawki, M. B., Nicolas, F., Lançon, C. ... Guedj, E. (2020 Jun). Involvement of the cerebellum in EMDR efficiency: a metabolic connectivity PET study in PTSD. Eur $J$ Psychotraumatol, 29;11((1), 1767986

53. Teng, C., Zhou, J., Ma, H., Tan, Y., Wu, X., Guan, C. ... Zhang, N. (2018). Abnormal resting state activity of left middle occipital gyrus and its functional connectivity in female patients with major depressive disorder.BMC psychiatry, 18(1), 370(1-9). 


\section{Tables}

Table 1: Demographics and clinical characteristics of the healthy controls, PTSD patients and TEC group

\begin{tabular}{|c|c|c|c|c|}
\hline PTSD $(n=27)$ & & TEC $(n=33)$ & $\mathrm{HC}(\mathrm{n}=30)$ & $p$ Value \\
\hline Gender (males/females) & $7 / 20$ & $7 / 26$ & $7 / 23$ & $.912^{*}$ \\
\hline Age (year) & $48.4 \pm 10.3$ & $48.5 \pm 7.5$ & $49.9 \pm 6.1$ & $.729^{\star \star}$ \\
\hline Education (year) & $6.4 \pm 3.4$ & $7.0 \pm 3.4$ & $9.7 \pm 3.3$ & $<0.001^{\star \star}$ \\
\hline $\begin{array}{l}\text { Days after } \\
\text { the disaster to examination }\end{array}$ & $105.5 \pm 9.5$ & $118.0 \pm 10.0$ & $125.8 \pm 1.0$ & $<0.001^{\star \star}$ \\
\hline SAS score & $65.8 \pm 13.3$ & $41.3 \pm 8.1$ & $36.0 \pm 5.5$ & $<0.001^{\star \star}$ \\
\hline SDS score & $69.6 \pm 13.2$ & $41.3 \pm 9.1$ & $33.5 \pm 7.2$ & $<0.001^{\star \star}$ \\
\hline $\begin{array}{l}\text { PCL score } \\
\text { CAPS total score }\end{array}$ & $\begin{array}{l}53.7 \pm 8.5 \quad 78.2 \pm 19.3 \\
24.52 \pm 7.27 \quad 28.07 \pm 8.26 \\
25.59 \pm 6.92\end{array}$ & $28.9 \pm 5.4$ & & $<0.001^{\star \star \star}$ \\
\hline \multicolumn{5}{|l|}{ Intrusion } \\
\hline \multicolumn{5}{|l|}{ Avoidance } \\
\hline Hypervigilance & & & & \\
\hline
\end{tabular}

Presented data are means \pm standard deviations.

Abbreviations: PTSD, post-traumatic stress disorder; SAS, Self-Rating Anxiety Scale; SDS, SelfRating Depression Scale; PCL, PTSD Checklist; CAPS, Clinician- Administered PTSD Scale; HC, healthy control; TEC, trauma-exposed control.

*P value obtained with chi-square test.

**P value obtained with one-way analysis of variance.

***P value obtained with independent $t$ test for continuous variables.

Table 2: Spearman correlational analysis between clinical scores and mean dwell time, number of transitions and fractional windows 
SAS

SDS PCL

CAPS Intrusion Total

$\begin{array}{lllllllll}\text { Dwell Time } & r & 0.201 & 0.198 & 0.089 & 0.327 & 0.322 & 0.118 & 0.439\end{array}$

State I
p
$0.043^{*}$
$0.046^{*} \quad 0.66$
0.096
0.101
0.557
$0.022^{*}$

Dwell Time

$-0.173-0.221$

$-0.143$

$-0.168$

$-0.176$

0

$-0.287$

State II
p $\quad 0.082$
$0.027^{*}$
$\begin{array}{lll}0.478 & 0.403 & 0.381\end{array}$
0.999
0.146

$\begin{array}{lllllllll}\text { Number of } & r & -0.197 & -0.186 & -0.069 & -0.344 & -0.305 & -0.163 & -0.449\end{array}$

Transitions

$\begin{array}{llllllll}p & 0.053 & 0.067 & 0.731 & 0.079 & 0.122 & 0.415 & 0.019^{*}\end{array}$

Fractional Windows

$\begin{array}{llllllll}r & 0.174 & 0.196 & 0.115 & 0.173 & 0.176 & 0.02 & 0.278\end{array}$
p $\quad 0.081$
$0.049^{*}$
$0.567 \quad 0.388$
0.379
0.923
0.16

\section{Figures}
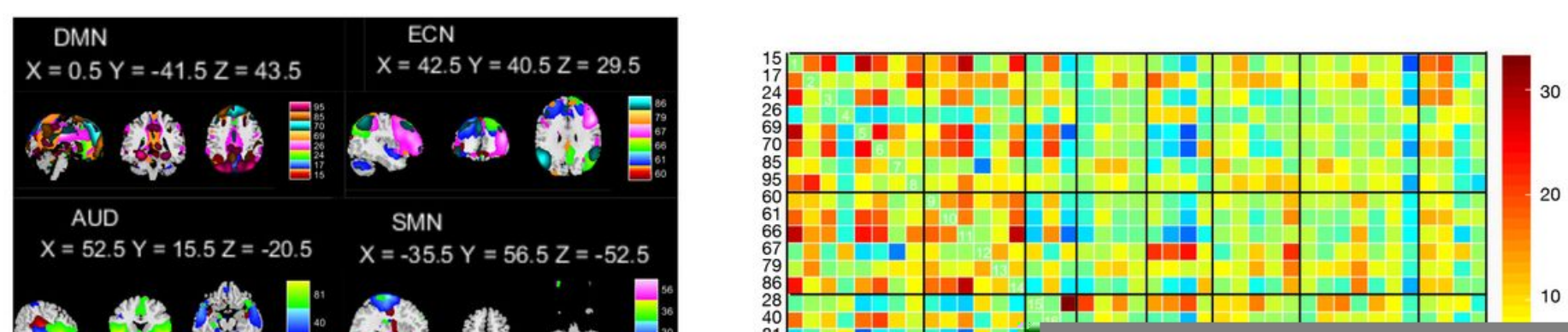

\section{Figure 1}

Independent components $(n=34)$ identified by group independent component analysis. DMN: Default Mode Network; ECN: Executive Control Network; AUD: Auditory Network; SMN: Sensory Motor Network; 

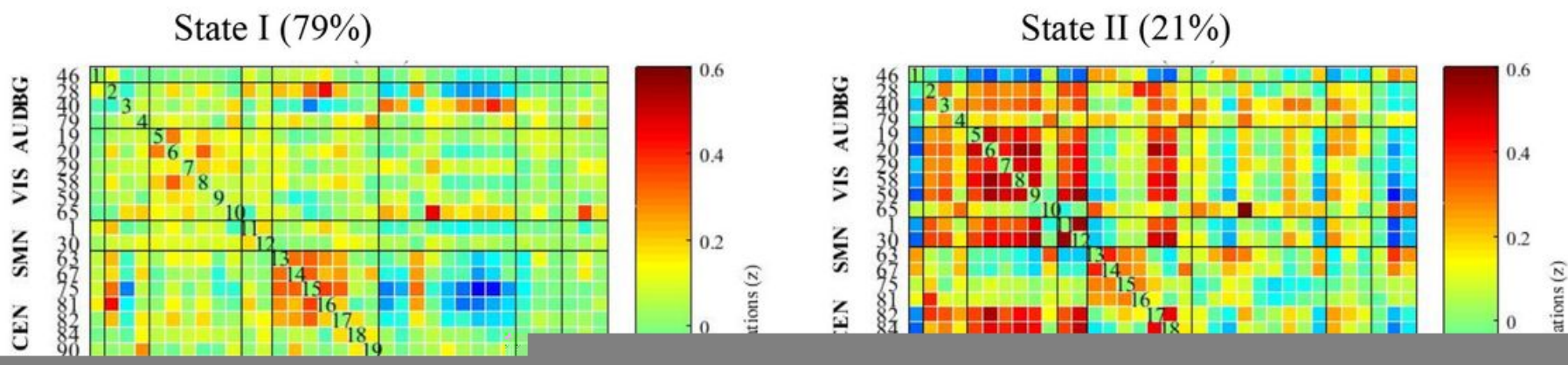

\section{Figure 2}

Results of the clustering analysis for each state. Bright colors indicated positive connections. Cool colors indicated negative connections. On average, the three groups spent $79 \%$ of time in State I, which was more segregated and less strongly interconnected, and $21 \%$ of time in State II, which was more strongly interconnected and had higher connectivity strength.
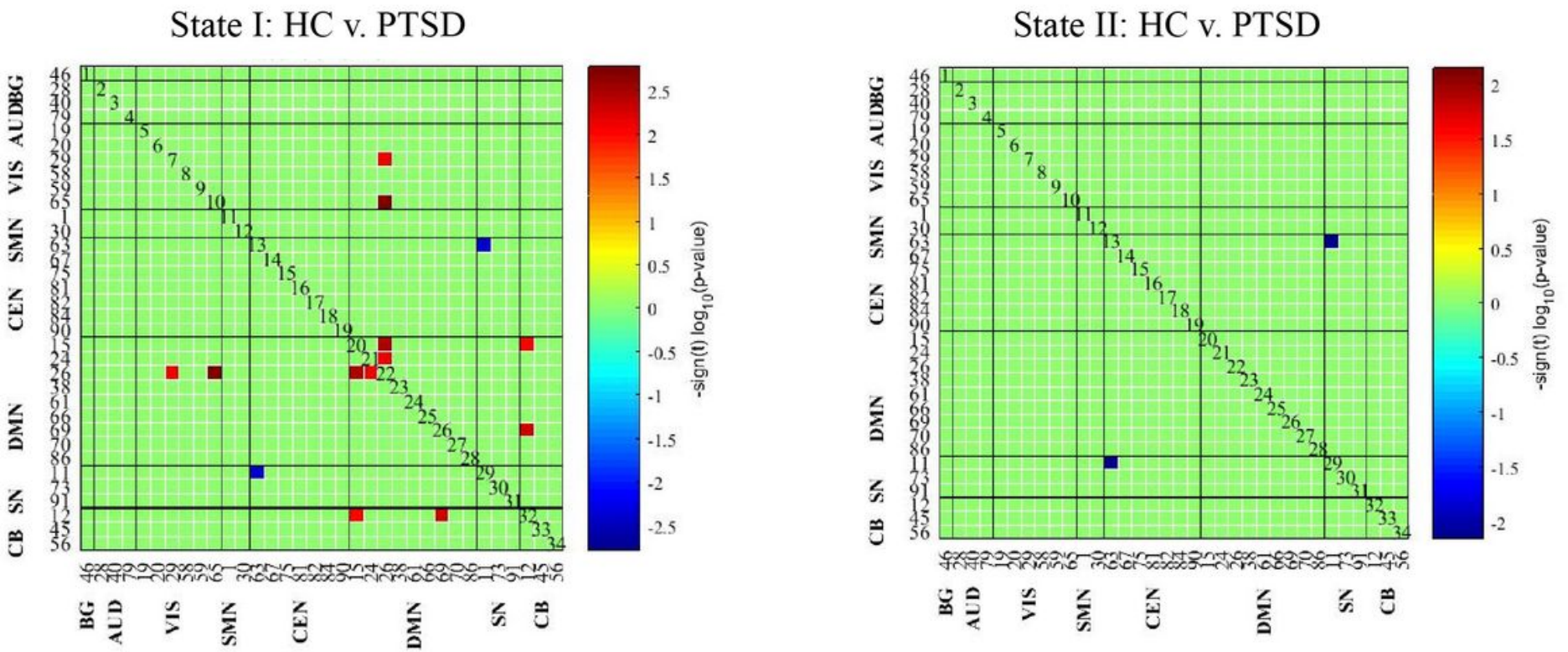

Figure 3 
Two sample t-test results of group HC v. group PTSD. Bright colors indicated stronger connectivity strength of HC compared with PTSD. Cool colors indicated weaker connectivity strength of HC compared with PTSD.

A

\section{Fractional Windows}

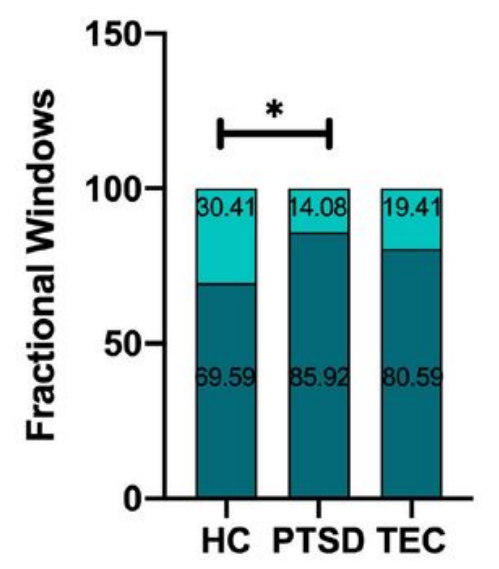

$\mathrm{C}$

\section{Number of Transitions}

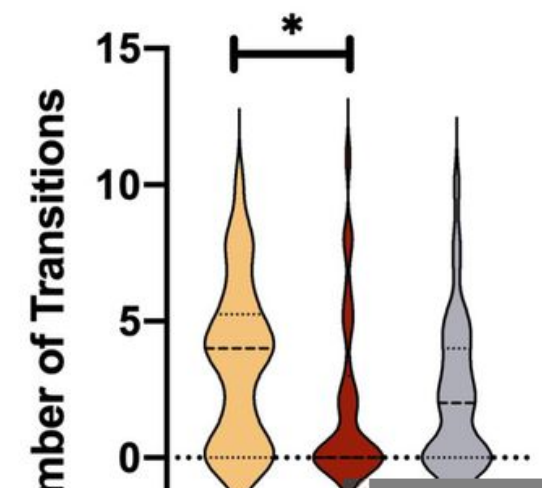

B

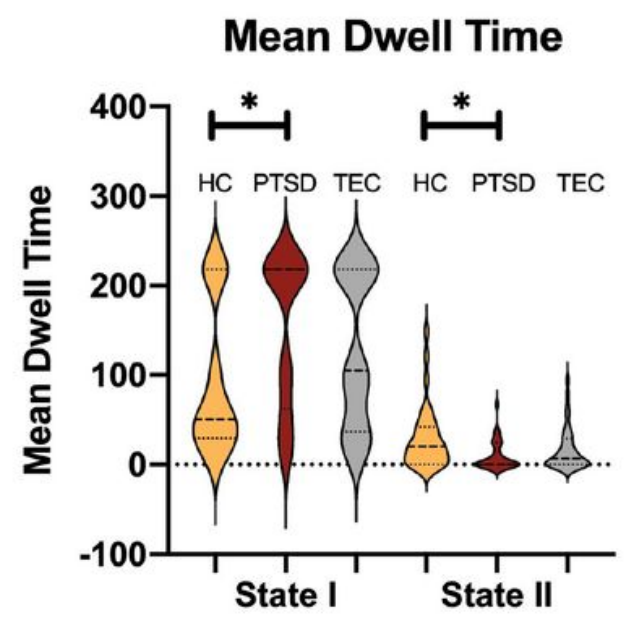

\section{Figure 4}


State I occurred more frequently for all three groups of participants. In addition, compared to control group, State I occurred significantly more frequent in PTSD group $(p=0.026)$. Meanwhile, it was observed that there was more frequent occurrence in State I for TEC group compared to control group, but PTSD had the most frequent occurrence in State I among the three groups.

\section{Supplementary Files}

This is a list of supplementary files associated with this preprint. Click to download.

- 1657317173667707BIBChecklist.docx

- 1657318173667707BIBAuthorDeclarationForm.docx 